\title{
TNF-a alters the inflammatory secretion profile of human first trimester placenta
}

\author{
Monika Siwetz ${ }^{1}$, Astrid Blaschitz ${ }^{1}$, Amin El-Heliebi ${ }^{1}$, Ursula Hiden², Gernot Desoye ${ }^{2}$, Berthold Huppertz ${ }^{1}$ and \\ Martin Gauster ${ }^{1}$
}

Implantation and subsequent placental development depend on a well-orchestrated interaction between fetal and maternal tissues, involving a fine balanced synergistic cross-talk of inflammatory and immune-modulating factors. Tumor necrosis factor (TNF)- $a$ has been increasingly recognized as pivotal factor for successful pregnancy, although high maternal TNF- $a$ levels are associated with a number of adverse pregnancy conditions including gestational hypertension and gestational diabetes mellitus. This study describes effects of exogenously applied TNF-a, mimicking increased maternal TNF- $a$ levels, on the secretion profile of inflammation associated factors in human first trimester villous placenta. Conditioned culture media from first trimester villous placental explants were analyzed by inflammation antibody arrays and ELISA after $48 \mathrm{~h}$ culture in the presence or absence of TNF- $a$. Inflammation antibody arrays identified interleukin (IL)-6, IL-8, chemokine (C-C motif) ligand 2 (CCL2), CCL4, and granulocyte-macrophage colony-stimulating factor (GM-CSF) as the most abundantly secreted inflammation-associated factors under basal culture conditions. In the presence of TNF- $a$, secretion of GM-CSF, CCL5, and IL-10 increased, whereas IL- 4 and macrophage CSF levels decreased compared with controls. ELISA analysis verified antibody arrays by showing significantly increased synthesis and release of GM-CSF and CCL5 by placental explants in response to TNF- $a$. Immunohistochemistry localized GM-CSF in the villous trophoblast compartment, whereas CCL5 was detected in maternal platelets adhering to perivillous fibrin deposits on the villous surface. mRNA-based in situ padlock probe approach localized GM-CSF and CCL5 transcripts in the villous trophoblast layer and the villous stroma. Results from this study suggest that the inflammatory secretion profile of human first trimester placenta shifts towards increased levels of GM-CSF, CCL5, and IL10 in response to elevated maternal TNF- $a$ levels, whereas IL- 6 and IL- 8 remain unaffected. This shift may represent a protective mechanism by human first trimester villous placenta to sustain trophoblast function and dampen inflammatory processes in the intervillous space.

Laboratory Investigation (2016) 96, 428-438; doi:10.1038/labinvest.2015.159; published online 11 January 2016

Implantation and subsequent placenta development are mandatory steps for successful human pregnancy and depend on a well-orchestrated interaction between fetal and maternal tissues. Fetal-maternal interaction involves a fine balanced synergistic cross-talk of inflammaory and immune-modulating factors to allow maternal immune adaption and tolerance of the semiallogeneic fetus at the one hand, whereas maternal immune functions need to be maintained to fight off infections on the other hand. Various concepts and paradigms have been suggested trying to explain how the maternal immune system is modulated to guarantee a viable pregnancy. One of the proposed paradigms is based on studies by Wegmann et al, ${ }^{1}$ describing a shift from an inflammatory T-helper 1 (Th1) cytokine profile to a rather anti-inflammatory T-helper 2 (Th2) profile. Meanwhile, the Th1/Th2 paradigm is considered an oversimplified representation of a complex immune network, which recently has been suggested to adapt to each stage of pregnancy and being characterized by unique inflammatory environments. ${ }^{2}$ Accordingly, the first and very early second trimester of pregnancy are characterized by a Th1 proinflammatory environment as a consequence of implantation and placentation processes. The second trimester represents a Th2 anti-inflammatory environment, enabling rapid fetal growth and development. Finally, the third trimester is characterized by a recurrence of inflammation preparing for parturition. With the shift from the Th1/Th2 paradigm toward new

${ }^{1}$ Institute of Cell Biology, Histology and Embryology, Medical University Graz, Graz, Austria and ${ }^{2}$ Department of Obstetrics and Gynaecology, Medical University Graz, Graz, Austria

Correspondence: Assistant Professor M Gauster, PhD, Institute of Cell Biology, Histology and Embryology, Medical University Graz, Harrachgasse 21/NI, Graz 8010, Austria. E-mail: martin.gauster@medunigraz.at

Received 18 August 2015; revised 23 September 2015; accepted 12 October 2015 
concepts, proinflammatory Th1 cytokines such as tumor necrosis factor (TNF)- $\alpha$ and interferon (IFN)- $\gamma$ have become increasingly recognized as pivotal factors for successful pregnancy. ${ }^{3,4}$ Thus, depending on every single stage of pregnancy, a combination of modulating signals and responses between the maternal immune system and the placental trophoblast are dynamically adjusted for an overall cooperative status. ${ }^{5}$

For TNF- $\alpha$, longitudinal analysis of maternal proinflammatory cytokines show significantly increasing levels from early and mid-to-late pregnancy, with a further increase at postpartum. ${ }^{6,7}$ A number of variables, such as maternal body mass index (BMI) and age are controversially discussed to influence maternal TNF- $\alpha$ levels during pregnancy, whereas increased TNF- $\alpha$ is associated with a number of adverse pregnancy conditions including gestational hypertension and gestational diabetes mellitus (GDM) ${ }^{7,8}$ Elevated TNF- $\alpha$ levels during distressed pregnancies have been suggested to affect trophoblast biology including migratory activity, syncytialization, and endocrine function. ${ }^{9,10}$ Moreover, elevated TNF- $\alpha$ may influence the fetal-maternal cross-talk by provoking a shift in the secretory profile of placenta-derived immunemodulating factors, which in turn influences the activity of maternal immune cells. Indeed, trophoblast-derived factors can induce differentiation of peripheral blood monocytes into macrophages ${ }^{11}$ and enhance recruitment and differentiation of inducible regulatory T cells (Tregs). ${ }^{12}$ Thus, it is now well acknowledged that the placenta functions as an immunemodulating organ that regulates the immune responses of cells present both at the implantation site and systemically. ${ }^{13}$ Among different types of trophoblasts, the highly differentiated syncytiotrophoblast is an integral part of the placental barrier, covers all placental villous trees, and thus is directly exposed to maternal blood. Direct contact with maternal blood enables the syncytiotrophoblast to respond to maternal conditions by releasing inflammatory and immunemodulating factors into the maternal circulation. Thus, systemic pro-inflammatory stimuli such as elevated maternal TNF- $\alpha$ may in turn provoke an aberrant release of cytokines and chemokines by the syncytiotrophoblast, potentially augmenting this feedforward-feedback loop. A growing body of evidence indicates that metabolic/pro-inflammatory conditions program early placenta functions and growth in the first trimester of pregnancy long before any phenotypic changes become clinically apparent. ${ }^{14}$ Moreover, it is increasingly recognized that the placenta early in gestation directly affects fetal development by responding to the maternal environment. ${ }^{15}$ Despite the importance of understanding placental responses to a pro-inflammatory environment early in pregnancy, little is known about this. Thus, the question was addressed whether exogenously applied TNF- $\alpha$, mimicking increased maternal TNF- $\alpha$, is able to influence the secretion profile of inflammation-associated factors in human first trimester villous placenta.

\section{MATERIALS AND METHODS Human Placental Tissue Samples}

The study was approved by the ethical committee of the Medical University of Graz. First trimester placental tissues (mean gestational week: 9.6 \pm 1.7 ) were obtained with written informed consent from women (mean maternal age: $26.1 \pm 6.4$ years; mean BMI: 20.9 \pm 2.6 ) undergoing legal elective pregnancy terminations.

\section{Placental Explant Culture}

Placental villous tissue from human first trimester $(n=8)$ was rinsed in buffered saline and dissected into small pieces of $\sim 5 \mathrm{mg}$ moist mass. Placental explants were cultured in 12-well dishes (nunc, Thermo Scientific, Roskilde, Denmark) and $2 \mathrm{ml} /$ well DMEM/F12 (1:1, Gibco, Invitrogen, Paisley, UK) supplemented with 10\% FCS, penicillin/streptomycin, amphotericin $\mathrm{B}$, and L-glutamine in a hypoxic workstation (BioSpherix, Redfield, NY, USA) under $2.5 \%$ oxygen at $37^{\circ} \mathrm{C}$ for $48 \mathrm{~h}$. For treatments, culture medium was supplemented with recombinant human TNF- $\alpha$ (Peprotech, Rocky Hill, NJ, USA) at indicated working concentrations. Cultivation of explants in complete culture medium without cytokines served as controls. After incubation, conditioned culture media were collected and spun down at $1500 \mathrm{~g}$ and $4{ }^{\circ} \mathrm{C}$ for $5 \mathrm{~min}$, to remove any cellular debris. Supernatants were aliquoted and stored at $-80^{\circ} \mathrm{C}$ until use. Placental explants were homogenized in RIPA buffer (Sigma-Aldrich, St Louis, MO, USA) including Protease Inhibitor Cocktail (Roche Diagnostics, Mannheim, Germany) using a tissue homogenizer. Homogenates were centrifuged at $8000 \mathrm{~g}$ and $4{ }^{\circ} \mathrm{C}$ for $10 \mathrm{~min}$. Concentration of total tissue protein was determined in homogenates according to Lowry method.

\section{Analysis of Placental Explant Viability}

Effects of TNF- $\alpha$ treatment on viability of placental explants was evaluated after culture by measurement of released lactate dehydrogenase (LDH) activity in culture supernatants using LDH Cytotoxicity Detection Kit (Takara Bio, Eubio, Vienna, Austria) according to the manufacturer's protocol. Obtained absorbance values were normalized to total protein of respective explant homogenates.

\section{Inflammation Antibody Array}

Secretion of inflammation-associated proteins by human first trimester placental explants was determined using a membrane based Human Inflammation Antibody Array (C3 Series, RayBiotech, Norcross, GA, USA), which consists of membranes for semi-quantitative detection of 40 human proteins involved in inflammation. Conditioned culture media of placental explants were proportionately pooled using aliquots according to total protein concentrations of respective explants. Pooled conditioned culture media from explants incubated with TNF- $\alpha$ were subjected to membrane arrays according to the manufacturer's instructions. Pooled conditioned culture media from explants incubated without 
TNF- $\alpha$ served as control. Chemiluminescent imaging was performed using the FluorChemQ System (Alpha Innotech, Cell Bioscienes, Santa Clara, CA, USA) and signal densities were analyzed with AlphaView software version 3.4.0. Complete culture medium alone was cultured in parallel to placental explants and values obtained from membrane array were subtracted from those of conditioned culture media.

\section{Determination of GM-CSF, CCL5, and IL-10}

Granulocyte-macrophage colony-stimulating factor (GM-CSF), chemokine (C-C motif) ligand 5 (CCL5), and interleukin (IL)-10 were measured in explant homogenates and corresponding conditioned culture media using quantitative sandwich enzyme immunoassays (Human GM-CSF, Human CCL5/regulated on activation, normal $\mathrm{T}$ cell expressed and secreted (RANTES), and Human IL-10 Quantikine ELISA, R\&D Systems, Minneapolis, MN, USA). Clear supernatants from conditioned culture media or placental explant homogenates were subjected to immunoassays according to the manufacturer's instruction. Complete culture medium incubated without explants and RIPA buffer served as blank for measurements in conditioned supernatants and tissue homogenates, respectively. Samples were measured in duplicates and obtained concentrations normalized to total tissue protein.

\section{Immunohistochemistry}

Formalin-fixed and paraffin-embedded (FFPE) placental tissue sections $(5 \mu \mathrm{m})$ were mounted on Superfrost Plus slides (Menzel/Thermo Fisher Scientific, Braunschweig, Germany). After deparaffinization, slides were subjected to antigen retrieval (AGR) in respective AGR buffers and a decloaking chamber (Biocare Medical, Sanova, Vienna, Austria) for $7 \mathrm{~min}$ at $120^{\circ} \mathrm{C}$. Sections were immunostained using the UltraVision Large Volume Detection System HRP Polymer Kit (Thermo Fisher Scientific, Runcorn, UK) as previously described. ${ }^{16}$ In brief, endogenous peroxidase was blocked using the UltraVision hydrogen peroxide block for $10 \mathrm{~min}$. After three washing steps with TBS including 0.05\% Tween 20 (TBS/T; Merck, Darmstadt, Germany), the background was blocked using Ultra Vision Protein Block for 5 min. Rabbit polyclonal antibodies against human GM-CSF (ab9741, Abcam, $2 \mu \mathrm{g} / \mathrm{ml}$; AGR buffer pH 9, Novocastra, Leica, Newcastle, UK) and human CCL5/RANTES (cat. no. PP1064P1, Acris, $2 \mu \mathrm{g} / \mathrm{ml}$; AGR buffer pH 6, Novocastra, Leica) were diluted in Antibody Diluent (Dako, Carpintera, CA, USA) and incubated on slides for $45 \mathrm{~min}$ at RT (GM-CSF) or $2 \mathrm{~h}$ at $4{ }^{\circ} \mathrm{C}$ (CCL5/RANTES). After three TBS/T washing steps, detection was achieved by incubation with the anti-rabbit HRP-labelled polymer system $(15 \mathrm{~min})$ and 3-amino-9-ethylcarbacole (Thermo Scientific, Runcorn, UK) according to the manufacturer's instructions. Nuclei were stained with hemalaun and slides were mounted with Kaiser's glycerol gelatine (Merck). For negative controls, slides were incubated with Negative Control for Rabbit IgG Ab-1
(NeoMarkers/Thermo Scientific) at the same concentration as mentioned above. Human tonsil from the archive of the Institute of Cell Biology, Histology, and Embryology, Medical University Graz, served as positive control for GM-CSF staining and was processed as described above. FFPE platelets obtained from healthy donors were used as positive control for CCL5 staining. Identity of platelets was confirmed by staining with rabbit polyclonal anti-human CD42b antibody (Proteintech, $5 \mu \mathrm{g} / \mathrm{ml}$, AGR buffer $\mathrm{pH}$ 9). For this purpose, platelet-rich plasma $(500 \mu \mathrm{l})$ was fixed in formalin at RT overnight. Thereafter, formalin-fixed platelets were washed in buffered saline and incubated with $5 \%$ gelatin for $45 \mathrm{~min}$ at $37^{\circ} \mathrm{C}$. Gelatin-embedded platelet pellets jelled at $4{ }^{\circ} \mathrm{C}$ and underwent another formalin fixation step. Fixed gelatinembedded platelets were subsequently embedded in paraffin by standard procedure and stained as described above.

\section{In Situ Detection of mRNA Transcripts by Padlock Probe Approach}

FFPE human first trimester placenta sections $(5 \mu \mathrm{m})$ were mounted on Superfrost Plus slides and pretreated for in situ experiments as previously described. ${ }^{17}$ Briefly, the tissue sections were deparaffinized, followed by a permeabilization in $2 \mathrm{mg} / \mathrm{ml}$ pepsin in $0.1 \mathrm{M} \mathrm{HCl}$ (Sigma) for $30 \mathrm{~min}$ at $37^{\circ} \mathrm{C}$. Slides were washed in DEPC- $\mathrm{H}_{2} \mathrm{O}$ and DEPC-PBS each for $2 \times 5 \mathrm{~min}$ at RT. After washing steps, sildes were dehydrated $\left(70,85\right.$, and $100 \% \mathrm{EtOH}, 1$ min each) and stored at $-80^{\circ} \mathrm{C}$ on use (up to 1 week). Oligonucleotides were designed using CLC Main Workbench software (CLC Bio Workbench Version 7.6, Qiagen, Venlo, The Netherlands) according to the guidelines published by Weibrecht et al. ${ }^{18}$ Sequences were retrieved from the National Center for Biotechnology Information with the GenBank accession numbers NM_002985.2 (CCL5) and NM_000758.3 (CSF2 and GM-CSF). Padlock probes were ordered 5'-phosphorylated (Integrated DNA Technologies, Coralville, IA, USA). LNA primers were purchased from Exiqon (Vedbaek, Denmark) and detection probes were purchased from Biomers (Ulm, Germany). Oligonucleotide sequences are shown in Table 1.

In situ reactions were performed with slight modifications as previously described. ${ }^{18}$ In detail, reactions were performed in secure seals hybridization chambers (Sigma) with a volume of $50 \mu$ l. Reverse transcription was performed with $5 \mathrm{U} / \mu \mathrm{l}$ Transcriptme Reverse Transcriptase (DNA-Gdansk, Gdansk, Poland), $1 \mu \mathrm{M}$ LNA primer each (Exiqon), $1 \mathrm{U} / \mu$ l RiboLock RNase inhibitor (Thermo Fisher Scientific, Waltham, MA, USA), $0.5 \mathrm{mM}$ dNTPs (Thermo Fisher Scientific), and $0.2 \mu \mathrm{g} /$ $\mu$ BSA (NEB, Ipswich, MA, USA) in the RT Reaction Buffer (Gdansk) (all units are displayed as final concentrations). Slides were incubated for $3 \mathrm{~h}$ at $45^{\circ} \mathrm{C}$ in a humid chamber. Directly after incubation, the reaction mix was removed by pipetting and tissue was postfixed with 3\% formaldehyde (Sigma) in DEPC-PBS for $10 \mathrm{~min}$. After postfixation, slides were washed twice with DEPC-PBS-T (DEPC-PBS with $0.05 \%$ Tween-20 (Sigma)) for 2 min each and forwarded to 
Sequences $\left(5^{\prime}-3^{\prime}\right)$

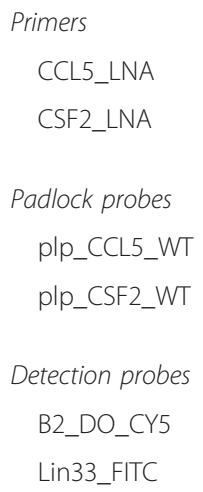

+ , the following base is LNA modified.

Underlined, target complement sequence.

Bold, detection probe complement sequence.

CY5 and FITC are fluorescent labels.

RNase $\mathrm{H}$ digestion and padlock probe ligation. The reaction mix consist of $0.5 \mathrm{U} / \mu \mathrm{l}$ Ampligase (Epicentre, Illumina, Madison, WI, USA), $0.4 \mathrm{U} / \mu \mathrm{l}$ RNase $\mathrm{H}$ (Thermo Fisher Scientific), $0.2 \mu \mathrm{g} / \mu \mathrm{l}$ BSA, $0.1 \mu \mathrm{m}$ of each padlock probe (Integrated DNA Technologies), $50 \mathrm{mM} \mathrm{KCl}$, and $20 \%$ formamide (Sigma) in Ampligase buffer. The samples were incubated for $30 \mathrm{~min}$ at $37^{\circ} \mathrm{C}$ and $45 \mathrm{~min}$ at $45^{\circ} \mathrm{C}$ in a humid chamber. After three washing steps $(1 \times$ SSC-Tween and $2 \times$ DEPC-PBS-T, each $5 \mathrm{~min}$ ), rolling-circle amplification was carried out with $1 \mathrm{U} / \mu \mathrm{l}$ phi29 DNA polymerase (Thermo Fisher Scientific), $0.25 \mathrm{mM}$ dNTPs (Thermo Fisher Scientific), $0.2 \mu \mathrm{g} / \mu \mathrm{l} \mathrm{BSA}$, and 5\% glycerol (Sigma) in phi29 buffer. Slides were incubated overnight at $37^{\circ} \mathrm{C}$ in a humid chamber. After rolling-circle amplification, slides were washed twice with DEPC-PBS-T and rolling-circle products were visualized with the corresponding detection probes as follows: $0.1 \mu \mathrm{M}$ of each detection probe (Biomers) in $2 \times$ SSC and $20 \%$ formamide for $30 \mathrm{~min}$ at $37^{\circ} \mathrm{C}$ in a humid chamber protected from light. Slides were washed with DEPC-PBS-T and nuclei were counterstained with $5 \mathrm{mg} / \mathrm{ml}$ DAPI (Thermo Fisher Scientific) for $5 \mathrm{~min}$ at RT. After a final washing step with DEPC-PBS-T, slides were dehydrated with EtOH (70, 85, and $100 \%, 1 \mathrm{~min}$ each) and mounted with SlowFade Gold Antifade Mountant (Thermo Fisher Scientific). Slides were stored at $4{ }^{\circ} \mathrm{C}$ protected from light until image analysis. Images were captured using the Zeiss Observer.Z1 inverted microscope (Carl Zeiss, Oberkochen, Germany) with a $\times 40$ objective and the AxioVision software (Carl Zeiss, Version 4.8.2.0). Z-stacks were combined in one layer as a maximum intensity projection with ZEN 2012 black software (Carl Zeiss, Version 8.1). Brightness and contrast of each image were adjusted for better visualization with ZEN 2012 black software (Carl Zeiss). Each detected signal was verified to be positive by checking all other fluorescent channels, as false positive signals are typically visible in multiple wavelengths. ${ }^{18}$

\section{Statistical Analysis}

Data were analyzed using SigmaPlot 12.5 and are presented as means \pm s.e.m. Data were subjected to normality test (Shapiro-Wilk test) and equal variance test. In case of normally distributed data, differences between groups were tested using two-tailed $t$-test. Otherwise Mann-Whitney rank-sum test was applied. For multiple comparison procedure, one-way repeated-measures analysis of variance was followed by Holm-Sidak method, to isolate groups that differ from the others. A $P$-value $<0.05$ was considered statistically significant.

\section{RESULTS}

\section{Secretion of Inflammation-Associated Proteins by} Human First Trimester Placenta

Analysis of conditioned culture media from human first trimester placental explants showed considerable secretory activity for inflammation-associated proteins after $48 \mathrm{~h}$ culture. Among 40 analyzed inflammation-associated proteins, IL-6, IL-8, and CCL2, also referred to as monocyte chemotactic protein-1, were the most abundantly secreted molecules (Figure 1). Moreover, CCL4 (or macrophage inflammatory protein (MIP)-1 $\beta$ ), GM-CSF, CCL3 (or MIP-1 $\alpha$ ), IFN- $\gamma$ induced protein-10, IL-4, CCL5 (or RANTES), and IL-10 were secreted by human first trimester placental explants. In addition to chemokines and cytokines, tissue inhibitor of metalloproteinases-2, soluble cytokine receptors for TNF (sTNFR II) and IL-6 (IL-6 sR) were detected in supernatants from placental explant cultures. 


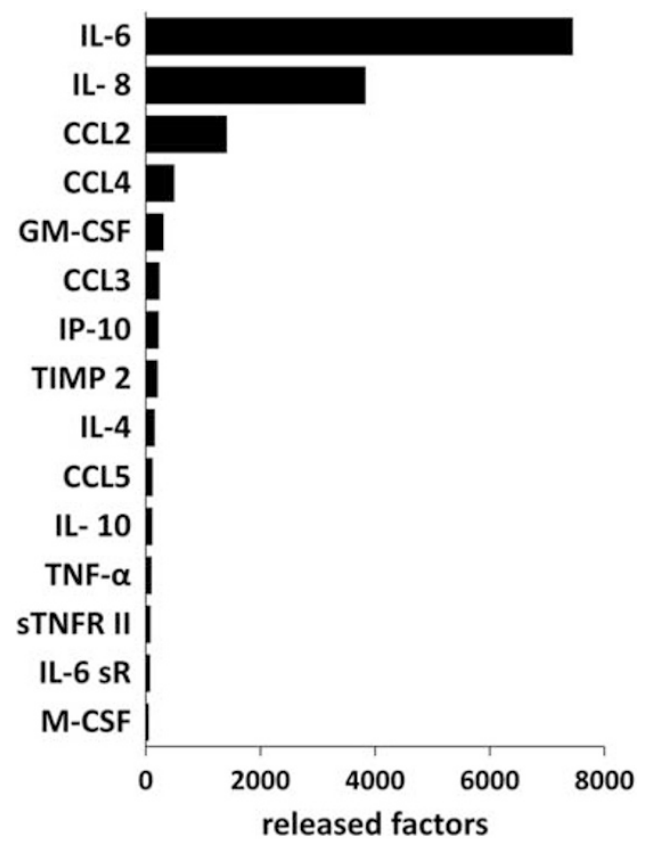

(a.u.)

Figure 1 Profile of inflammation associated proteins secreted by human first trimester placenta. Conditioned culture media from different human first trimester placental explants $(n=8)$ were pooled after $48 \mathrm{~h}$ culture and analyzed using a membrane-based antibody array for semiquantitative detection of 40 inflammation-associated proteins. Signal densities were analyzed by densitometry and normalized to the internal control. Data are given as arbitrary units (a.u.).

\section{TNF- $a$ Altered the Profile of Secreted Inflammation-} Associated Proteins by Human First Trimester Placenta As some pregnancy pathologies are associated with elevated circulating maternal TNF- $\alpha$, we next tested the effect of exogenous TNF- $\alpha$ on placental secretion of inflammationassociated proteins in human first trimester placental explant culture. For this purpose, placental explants were incubated in the presence or absence of TNF- $\alpha(10 \mathrm{ng} / \mathrm{ml})$ and conditioned culture media were analyzed for secreted inflammationassociated proteins after $48 \mathrm{~h}$. Accordingly, TNF- $\alpha$ increased the secretion of GM-CSF, CCL5, and IL-10 by $73 \%, 119 \%$, and $82 \%$, respectively, when compared with control. In contrast, secretion of IL-4 and macrophage colonystimulating factor was decreased by $45 \%$ and $36 \%$, respectively, in response to TNF- $\alpha$ (Figure 2). To ensure that observed effects were not due to TNF- $\alpha$-mediated cytotoxicity, the release of LDH into the culture medium was analyzed after culture and showed no significant difference between TNF- $\alpha$-treated explants and control (not shown).

Next, these results were verified for GM-CSF, CCL5, and IL-10 by complementary ELISA analysis of placental explant homogenates and corresponding conditioned culture media. Accordingly, GM-CSF levels in explant homogenates increased $1.20( \pm 0.35)$-fold and $2.07( \pm 0.44, P<0.05)$-fold when incubated in the presence of 1 and $10 \mathrm{ng} / \mathrm{ml} \mathrm{TNF}-\alpha$,

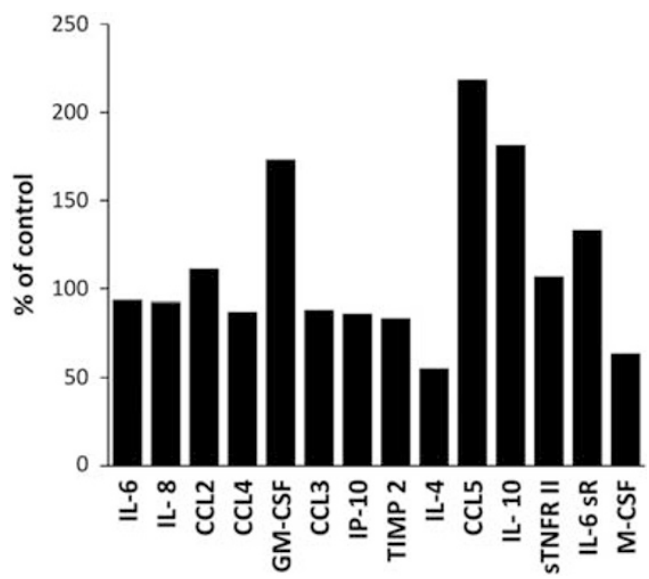

Figure 2 Secretion of inflammation-associated proteins by human first trimester placenta in response to tumor necrosis factor (TNF)- $a$. Human first trimester placental explants $(n=8)$ were incubated with or without recombinant TNF- $a(10 \mathrm{ng} / \mathrm{ml})$ for $48 \mathrm{~h}$. Conditioned culture media from placental explants were pooled and analyzed by an inflammation antibody array. Signal densities were analyzed by densitometry, normalized to the internal control, and compared between TNF- $a$-treated and -untreated explants. Data are given as \% of control.

respectively (Figure 3a). Secretion of GM-CSF significantly increased $2.17( \pm 0.89, P<0.05)$-fold in the presence of TNF- $\alpha$ at a concentration of $10 \mathrm{ng} / \mathrm{ml}$, whereas incubation at $1 \mathrm{ng} / \mathrm{ml}$ led to a nonsignificant decrease by $12 \%$ (Figure $3 \mathrm{~b}$ ). Similar to GM-CSF, CCL5 levels in explant homogenates were 1.23 $( \pm 0.20)$-fold and $1.89( \pm 0.34, P<0.01)$-fold increased in response to TNF- $\alpha$ at 1 and $10 \mathrm{ng} / \mathrm{ml}$, respectively, when compared with control after $48 \mathrm{~h}$ (Figure 3c). Likewise, levels of secreted CCL5 in corresponding culture supernatants increased $1.34( \pm 0.35)$-fold and $2.18( \pm 0.39, P<0.05)$-fold in the presence of TNF- $\alpha$ at 1 and $10 \mathrm{ng} / \mathrm{ml}$, respectively (Figure 3d). Levels of tissue-associated and -secreted IL-10 were remarkably lower when compared with GM-CSF and CCL5, but followed the same, albeit not significant, trend of upregulation in response to TNF- $\alpha$ (Figures $3 \mathrm{e}$ and $\mathrm{f}$ ).

\section{Released GM-CSF and CCL5 are of Fetal and Maternal Origin}

Although placental IL-10 expression has been shown in the villous trophoblast of first and second trimester, ${ }^{19}$ knowledge about the site of placental GM-CSF and CCL5 expression is rather unclear. For this reason, we next performed immunohistochemistry for GM-CSF and CCL5 in human first trimester placenta sections. Immunohistochemical staining showed intense GM-CSF expression in Hofbauer cells, fetal endothelium and some villous cytotrophoblasts, whereas the syncytiotrophoblast showed only very weak expression and villous stromal cells did not express GM-CSF. Interestingly, very intense vesicle-like GM-CSF staining was detected in apical regions of some cytotrophoblasts (Figure 4a). Human tonsil served as positive control and showed intense GM-CSF staining of macrophages embedded in lymphoid tissue 

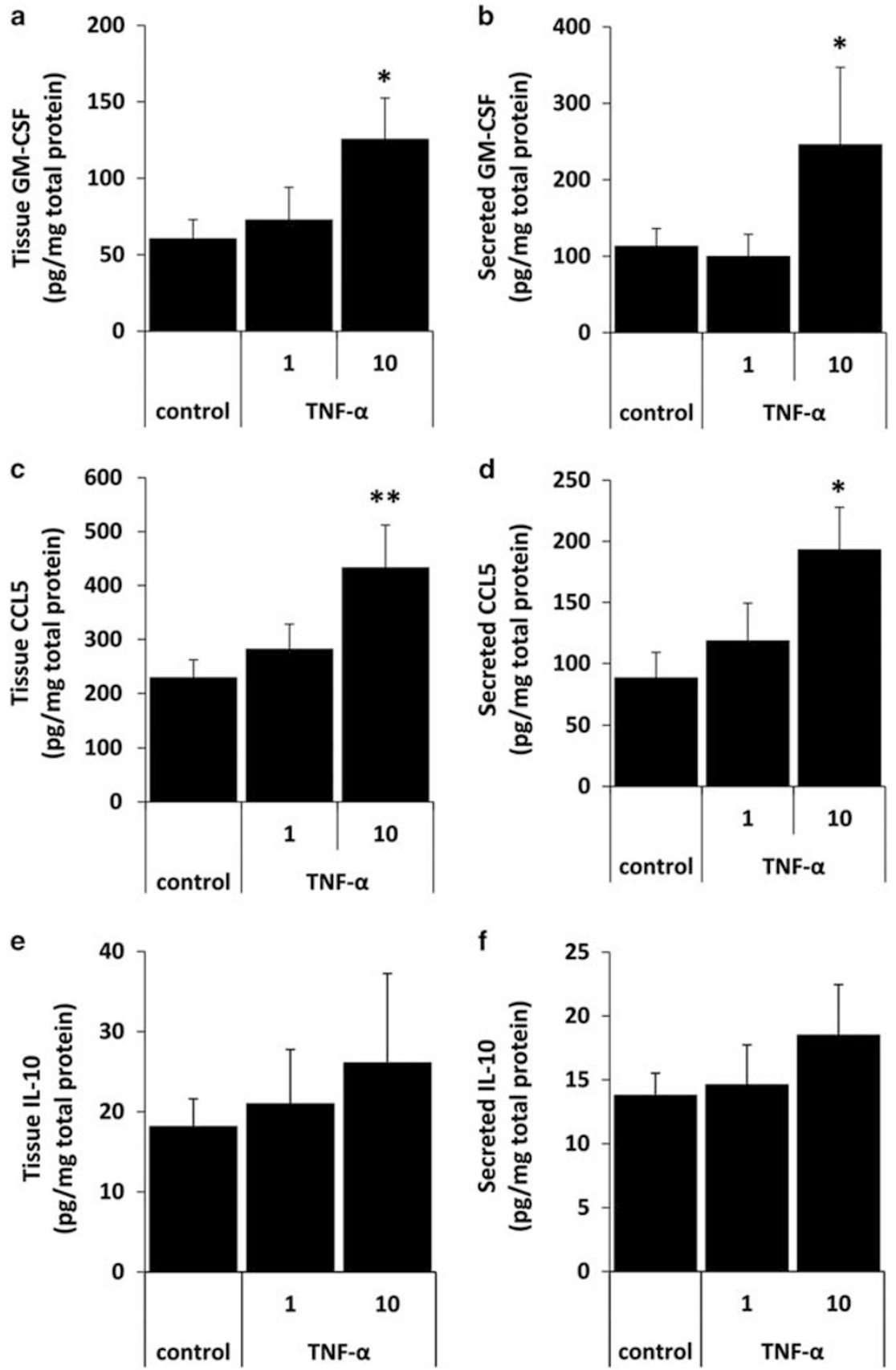

Figure 3 Secretion of granulocyte-macrophage colony-stimulating factor (GM-CSF), chemokine (C-C motif) ligand 5 (CCL5), and interleukin (IL)-10 by placental explants is increased in response to tumor necrosis factor (TNF)- $a$. Human first trimester placental explants $(n=8)$ were incubated with indicated concentrations of TNF- $a(\mathrm{ng} / \mathrm{ml})$ for $48 \mathrm{~h}$. After culture, explant homogenates and corresponding culture supernatants were analyzed by enzyme-linked immunosorbent assay (ELISA) and obtained values normalized to total tissue protein. Values are given as mean \pm s.e.m. ${ }^{*} P \leq 0.05$ and ${ }^{* *} P \leq 0.01$.

(Figure 4b) Immunohistochemistry for CCL5 showed neither staining of the villous trophoblast layer, Hofbauer cells, and fetal endothelium, nor the villous stroma. However, CCL5 staining was sporadically detected in leukocytes and fetal platelets within fetal blood vessels (not shown). Moreover, CCL5 staining was frequently detected associated with perivillous fibrin deposits on the villous surface (Figure 4c).
The small spot-like staining pattern suggested adhering maternal platelets accounting for the observed staining, which was confirmed on consecutive serial sections by staining for CD42b, a surface membrane protein of platelets (Figure 4d). FFPE platelets served as positive control and showed considerable CCL5 (Figure 4f) and CD42b (Figure 4h) staining, whereas GM-CSF was not detected (Figure $4 \mathrm{~g}$ ). 

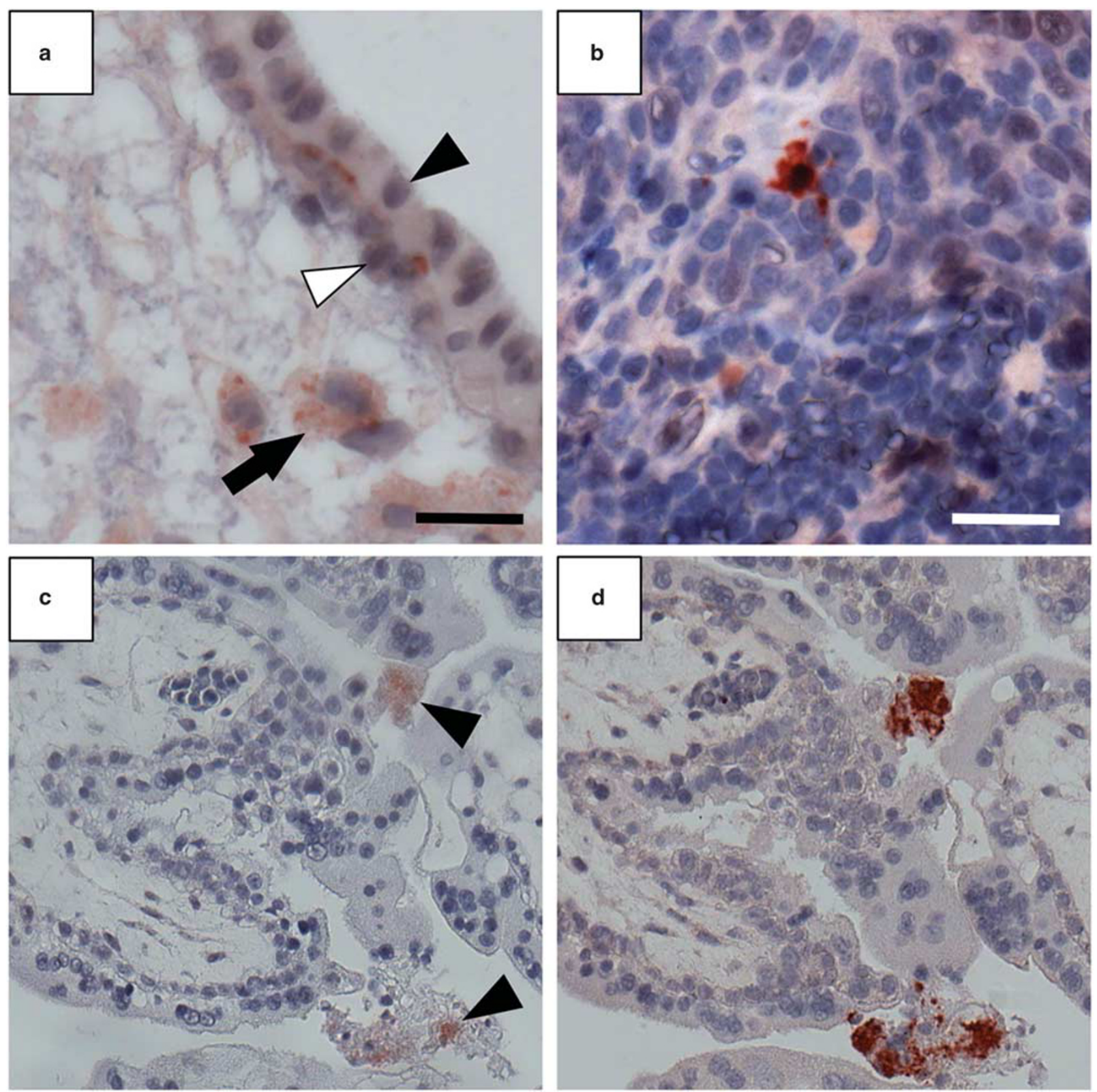

Figure 4 Immunohistochemical localization of granulocyte-macrophage colony-stimulating factor (GM-CSF) and chemokine (C-C motif) ligand 5 (CCL5) in human first trimester placenta. In first trimester placental villi, GM-CSF was detected in Hofbauer cells (arrow) and some villous cytotrophoblasts (open arrowhead), whereas the syncytiotrophoblast (arrowhead) showed only very weak staining. Intense vesicle-like GM-CSF staining was detected in apical regions of some cytotrophoblast (a). Human tonsil served as positive control and showed intense GM-CSF staining of macrophages (b). CCL5 was detected in maternal platelets adhering to perivillous fibrin deposits (c, arrowheads), whereas villous trophoblast, Hofbauer cells, fetal endothelium, and villous stroma were unstained. Maternal platelets were identified on consecutive serial sections by staining for CD42b (d). Incubation with negative control rabbit IgG revealed no staining (e). Formalin fixed and paraffin embedded (FFPE) human platelets showed intense CCL5 (f) staining, whereas GM-CSF was not detected (g). Identity of platelets was confirmed by CD42b (h) and negative control rabbit lgG revealed no staining (h, insert). Scale bars in $\mathbf{a}$ and $\mathbf{b}$ represent $20 \mu \mathrm{m}$ and those in $\mathbf{e}$ and $\mathbf{f}$ represent $50 \mu \mathrm{m}$.

\section{GM-CSF and CCL5 Transcripts are Detectable in the Villous Trophoblast}

In order to extend findings from immunohistochemistry, we performed the probably more sensitive mRNA-based in-situ padlock probe approach, to localize GM-CSF and CCL5 transcripts in human first trimester placenta sections. GM-CSF mRNA molecules were frequently detected in the villous trophoblast layer and the villous stroma (Figure 5a). Unlike GM-CSF, CCL5 transcripts were only sporadically detected in the villous trophoblast (Figure 5b) and 

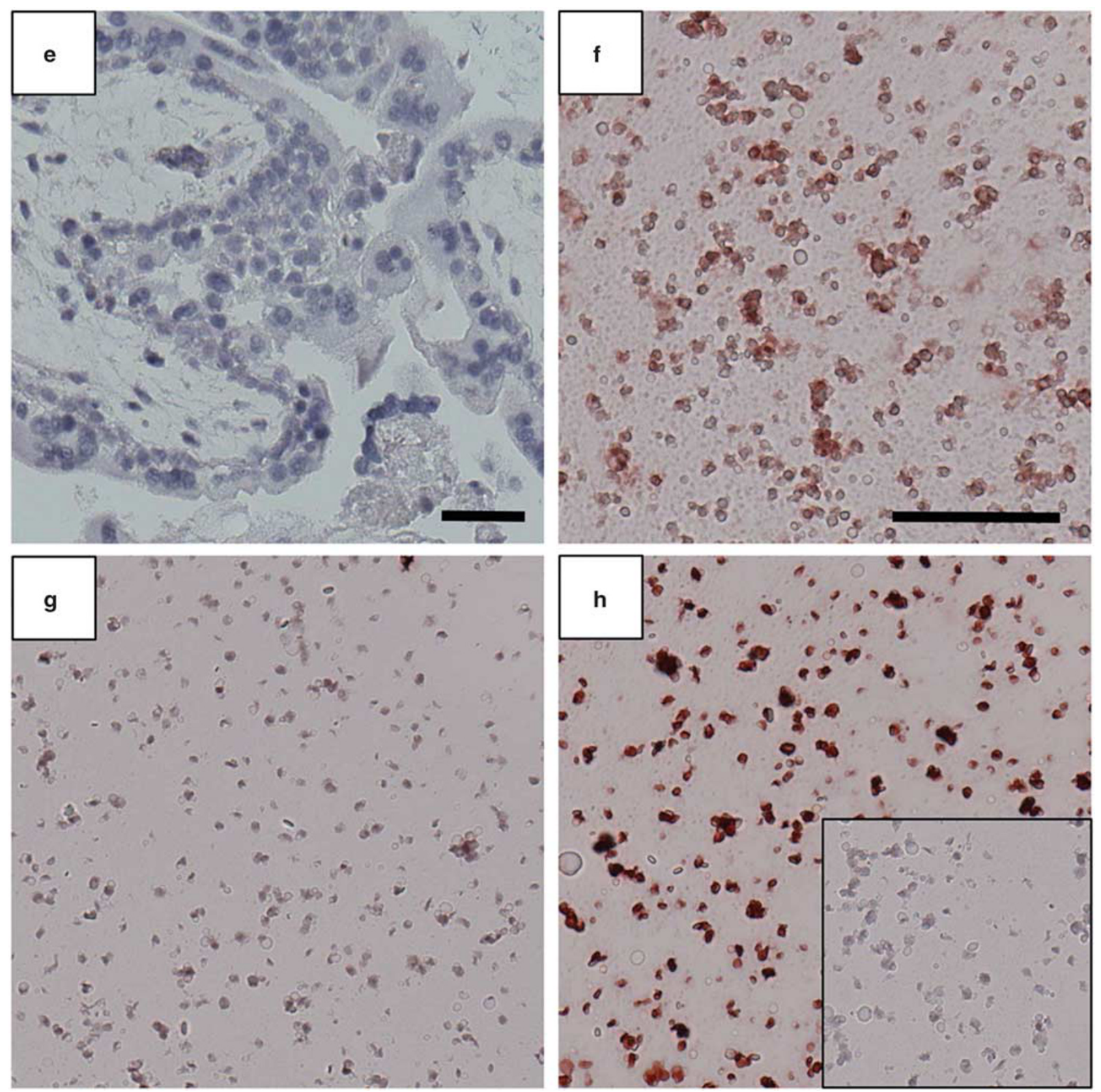

Figure 4 Continued.

occasionally in the villous stroma. The presence of focal signal spots at apical sites of the outer layer of nuclei as well as basal sites of the inner trophoblast layer suggested GM-CSF and CCL5 transcripts in both the syncytiotrophoblast and villous cytotrophoblast layer.

\section{DISCUSSION}

Human first trimester villous placenta releases a cocktail of inflammatory and immune-modulating factors, whose mixture considerably shifts in response to exogenous TNF$\alpha$. This shift is characterized by significantly increased release of GM-CSF and CCL5, which however seem to arise from different origin. Results from placental explant experiments suggest that both fetal- and maternal-derived factors are released from placental villi into the maternal circulation, in response to elevated maternal TNF- $\alpha$. This paradox is based on maternal platelets adhering to perivillous fibrin deposits, from where a bulk of platelet-derived factors such as CCL5, CCL3, chemokine (C-X-C motif) ligand 1 (CXCL1), CXCL4, CXCL5, and CXCL7 may be released into the intervillous space. The fact that CCL5 was detected in adhering maternal platelets, but was virtually absent in the villous trophoblast 

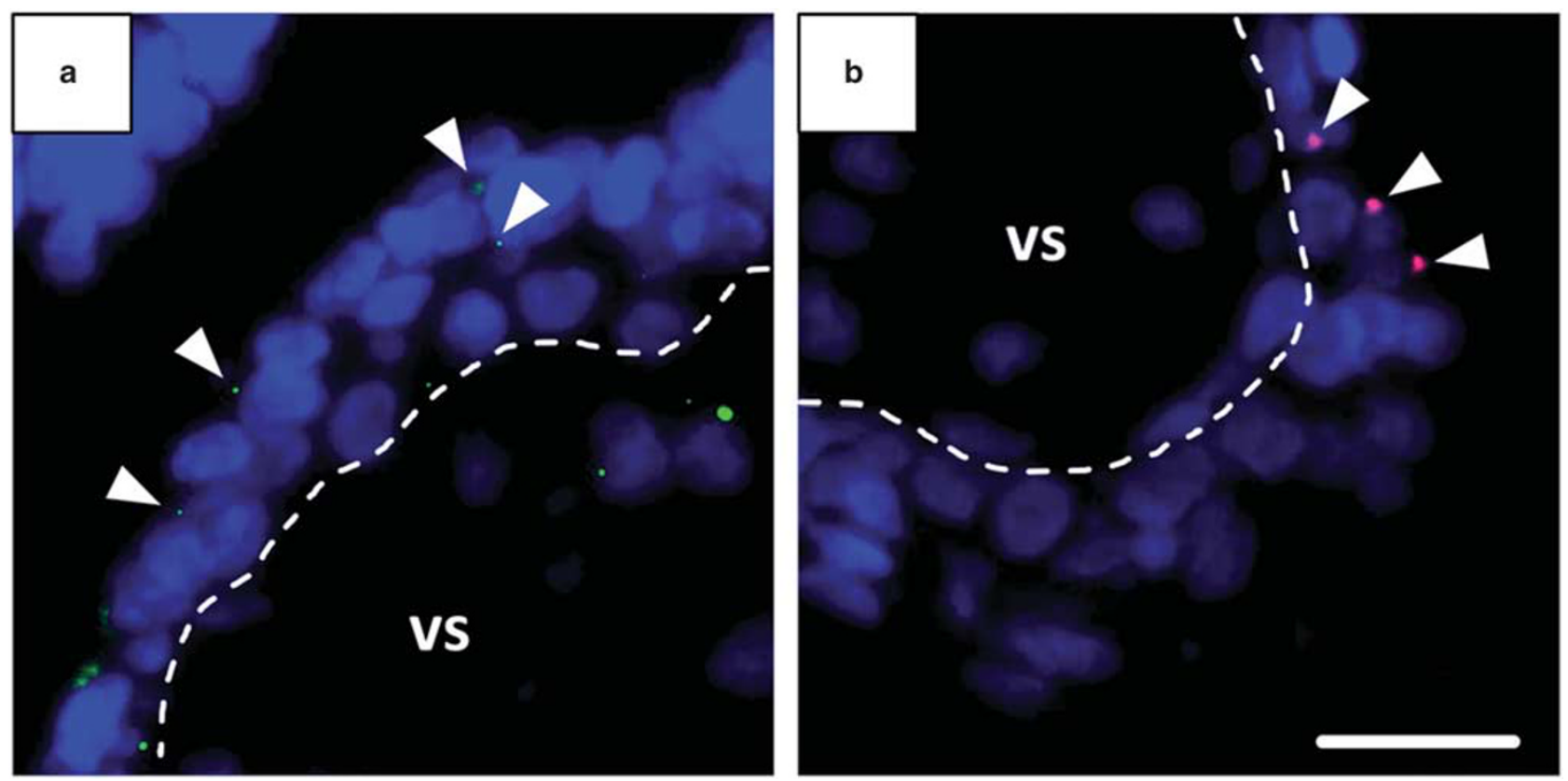

Figure 5 Localization of granulocyte-macrophage colony-stimulating factor (GM-CSF) and chemokine (C-C motif) ligand 5 (CCL5) transcripts in human first trimester placenta. GM-CSF transcripts were frequently localized in the villous trophoblast layer (arrowheads) and the villous stroma (vs) using in-situ padlock probe technology and green fluorescent detection probes (a). CCL5 transcripts were rarely detected in the villous trophoblast (arrowheads) and in the villus stroma using red fluorescent detection probes (b). Scale bar represents $20 \mu \mathrm{m}$.

layer, provides a plausible explanation for previous studies showing detectable CCL5 secretion levels in early and term placental villi, even though synthesis and release of this chemokine has not been detected in primary cytotrophoblast cultures. ${ }^{20,21}$ By complementary analysis, using the padlock probe approach, we detected CCL5 mRNA transcripts in the villous trophoblast compartment, suggesting that protein levels may probably have been too low to allow their detection by immunohistochemistry. In addition to platelets, also fibroblasts, mesangial cells, and epithelial cells express CCL5 on activation with TNF- $\alpha,{ }^{22}$ suggesting that villous trophoblast may contribute little amounts of CCL5 to the cocktail of released factors in response to inflammatory stimuli. Unlike villous trophoblast, which seems to only marginally express CCL5, trophoblast cell lines Swan 71 and HTR-8/SVneo clearly produce CCL5 (ref. 23) and, moreover, show increased CCL5 secretion in response to lipopolysaccharide (LPS) and TNF- $\alpha .{ }^{24,25}$ Although increased CCL5 release in response to inflammatory stimuli is in line with the present study, it remains an open question whether basal CCL5 production found in trophoblast cell lines is the result of their immortalization or reflects their rather extravillous phenotype.

In contrast to CCL5, GM-CSF is substantially produced in the villous trophoblast layer, from where it can be released into the maternal circulation. Thus, CCL5 mostly released from adhering maternal platelets together with trophoblastderived GM-CSF may act in concert to contribute to the inflammatory microenvironment of the intervillous space. There, both factors may interfere with either circulating maternal blood cells or the trophoblast. Although CCL5 may increase Tregs and induce apoptosis of maternal alloactivated $\mathrm{T}$ cells in favor of maternal tolerance, ${ }^{23} \mathrm{GM}$-CSF may act on the villous trophoblast in an autocrine manner. ${ }^{26}$ Indeed, GM-CSF receptor subunit GM-R $\alpha$ is present on first trimester cytotrophoblast, extravillous trophoblasts, and at lower levels on the syncytiotrophoblast. ${ }^{27}$ This way, GM-CSF is able to induce differentiation of villous cytotrophoblasts and to stimulate secretion of placental lactogen, human chorionic gonadotropin, ${ }^{28}$ and IL-10, ${ }^{29}$ which is considered to have a central role in suppressing the activities of pro-inflammatory Th1 cytokines. ${ }^{30,31}$ In the light of these observations, enhanced release of GM-CSF and CCL5 in response to elevated maternal TNF- $\alpha$ may be interpreted as fetal attempt to protect the villous placenta from exaggerated maternal inflammation. This assumption is supported by the trend of increased IL-10 release in TNF- $\alpha$-treated placental explants, which however was very low compared with CCL5 and GM-CSF levels. IL-10 is suggested to regulate cytotrophoblast activity in a paracrine and autocrine manner. ${ }^{32,33}$ Interestingly, administration of exogenous IL-10 significantly reduces TNF- $\alpha$ concentrations in conditioned culture medium from term placental explants cultured under $2 \%$ oxygen. ${ }^{34}$ Moreover, IL-10 induces extrathymic differentiation of peripheral $\mathrm{T}$ cells to become Tregs. Tregs induced by IL-10 are CD4+CD25Foxp3 - and are referred to as $\operatorname{Tr} 1$ cells, which suppress immune responses by secretion of IL-10..$^{35,36}$ Recent studies in mice suggest that Tregs, predominantly of thymic origin, are required before implantation, whereas 
thereafter at later stages of pregnancy, extrathymically differentiated Tregs contribute to the Treg pool in the periphery. ${ }^{37}$ Extrathymic generation of Tregs seems to be restricted to eutherian mammals and emerged during evolution as a mechanism mitigating the maternal-fetal allgenic conflict. ${ }^{38}$ Based on these observations, it is tempting to speculate that placenta-derived IL-10 contributes to extrathymic generation of Tregs, which have the capacity to actively inhibit proliferation and effector functions of other $\mathrm{T}$ cells and thereby have a key role in the new Th1/Th2/Th17 and Treg paradigm. ${ }^{39,40}$

As a note of caution, the effects of TNF- $\alpha$ stimulation may differ between primary trophoblasts and placental explants, where cells remain in their natural microenvironment and preparatory stress is reduced to a minimum. ${ }^{41,42}$ Moreover, TNF- $\alpha$ stimulation may have different effects in vitro compared with local effects of the cytokine in vivo, in particular at low doses. ${ }^{43}$ This speculation is based, eg, on angiogenesis studies showing predominantly anti-angiogeneic effects of TNF- $\alpha$ on in vitro-cultured endothelial cells, but pro-angiogeneic activities in vivo. ${ }^{44}$ Maternal TNF- $\alpha$ plasma levels range within the concentration of $\mathrm{pg} / \mathrm{ml}$ in healthy, pregnant women and are increased in GDM and preeclamptic women. ${ }^{45-47}$ In the present study, TNF- $\alpha$ at concentrations of 1 and $10 \mathrm{ng} / \mathrm{ml}$ were used, which are in fact higher than observed in vivo levels. However, used concentrations are in line with a number of other placental explant studies $9,10,48$ and may reflect microenvironmental concentrations in the intervillous space, where autocrine/paracrine acting TNF- $\alpha$ concentrations may be higher than systemic levels.

Among the cytokines and chemokines analyzed here, IL- 6 , IL-8, and CCL2 are the most abundantly secreted factors from human first trimester placental explants under basal culture conditions. Although largely considered as inflammatory factors, it is worth noting that IL-8 and CCL2 have recently been suggested to regulate a number of non-inflammatory functions in human early pregnancy. ${ }^{49}$ Both, IL-8 and CCL2 derived from human villous first trimester trophoblasts were shown to enhance multistep processes of angiogenesis such as permeability, migration, proliferation, and capillary tube formation of human endometrial microvascular endothelial cells. ${ }^{50}$ Although abundant IL-6 and IL-8 secretion under basal conditions is in good agreement with other studies, results from our explant experiments suggest that secretion of both IL-6 and IL-8 is not considerably affected by exogenous TNF- $\alpha$ in human first trimester placenta. Interestingly, IL-6 and IL-8 secretion by first trimester placental explants is also not affected by para-Nonylphenol, a ubiquitous environmental contaminant described to significantly increase placental secretion of inflammatory cytokines such as IFN- $\gamma$, IL- $1 \beta$, and TNF- $\alpha .{ }^{51}$ In contrast to data from the first trimester, data from term placental explant and syncytiotrophoblast cultures show increased IL-6 and IL-8 secretion in response to the inflammatory stimulus LPS. ${ }^{52-54}$ Thus, placental IL-6 and IL-8 response may be differentially regulated depending on gestational age and inflammatory stimulus. This contention may even apply for different trophoblast subtypes, as differential inflammatory response to LPS has been shown for trophoblast cell lines Jeg-3 and BeWo. ${ }^{55}$

In summary, we conclude that the inflammatory secretion profile of human first trimester placenta shifts toward increased levels of GM-CSF, CCL5, and IL10 in response to increased maternal TNF- $\alpha$ levels, whereas IL-6 and IL- 8 remain unaffected. This shift may represent a protective mechanism by the villous placenta to sustain trophoblast function and dampen inflammatory processes in the intervillous space.

\section{ACKNOWLEDGMENTS}

MG was supported by funds of the Oesterreichische Nationalbank (Oesterreichische Nationalbank, Anniversary Fund, project number: 16513) and the Austrian Science Fund (FWF): P23859-B19. AE-H was supported by the START funding program of the Medical University of Graz. We thank Andreas Glasner for recruiting first trimester placental tissue samples for this study.

\section{DISCLOSURE/CONFLICT OF INTEREST}

The authors declare no conflict of interest.

1. Wegmann TG, Lin H, Guilbert L et al. Bidirectional cytokine interactions in the maternal-fetal relationship: Is successful pregnancy a $\mathrm{TH} 2$ phenomenon? Immunol Today 1993;14:353-356.

2. Mor G, Cardenas I, Abrahams V et al. Inflammation and pregnancy: the role of the immune system at the implantation site. Ann N Y Acad Sci 2011;1221:80-87.

3. Hunt JS, Chen HL, Miller L. Tumor necrosis factors: pivotal components of pregnancy? Biol Reprod 1996;54:554-562.

4. Argiles JM, Carbo N, Lopez-Soriano FJ. TNF and pregnancy: the paradigm of a complex interaction. Cytokine Growth Factor Rev 1997;8:181-188.

5. Racicot K, Kwon JY, Aldo P et al. Understanding the complexity of the immune system during pregnancy. Am J Reprod Immunol 2014;72: 107-116.

6. Christian LM, Porter K. Longitudinal changes in serum proinflammatory markers across pregnancy and postpartum: effects of maternal body mass index. Cytokine 2014;70:134-140.

7. Pantham P, Aye IL, Powell TL. Inflammation in maternal obesity and gestational diabetes mellitus. Placenta 2015;36:709-715.

8. Peracoli JC, Rudge MV, Peracoli MT. Tumor necrosis factor-alpha in gestation and puerperium of women with gestational hypertension and pre-eclampsia. Am J Reprod Immunol 2007;57:177-185.

9. Bauer S, Pollheimer J, Hartmann J et al. Tumor necrosis factor-alpha inhibits trophoblast migration through elevation of plasminogen activator inhibitor-1 in first-trimester villous explant cultures. J Clin Endocrinol Metab 2004;89:812-822.

10. Leisser C, Saleh L, Haider S et al. Tumour necrosis factor-alpha impairs chorionic gonadotrophin beta-subunit expression and cell fusion of human villous cytotrophoblast. Mol Hum Reprod 2006;12:601-609.

11. Aldo PB, Racicot $K$, Craviero $V$ et al. Trophoblast induces monocyte differentiation into CD14+/CD16+ macrophages. Am J Reprod Immunol 2014;72:270-284.

12. Ramhorst $R$, Fraccaroli $L$, Aldo $P$ et al. Modulation and recruitment of inducible regulatory $\mathrm{T}$ cells by first trimester trophoblast cells. Am J Reprod Immunol 2012;67:17-27.

13. PrabhuDas M, Bonney $\mathrm{E}$, Caron $\mathrm{K}$ et al. Immune mechanisms at the maternal-fetal interface: Perspectives and challenges. Nat Immunol 2015;16:328-334.

14. Catalano P, deMouzon SH. Maternal obesity and metabolic risk to the offspring: why lifestyle interventions may have not achieved the desired outcomes. Int J Obes (Lond) 2015;39:642-649.

15. Desoye G, van Poppel M. The feto-placental dialogue and diabesity. Best Pract Res Clin Obstet Gynaecol 2015;29:15-23. 
16. Siwetz M, Dieber-Rotheneder M, Cervar-Zivkovic $M$ et al. Placental fractalkine is up-regulated in severe early-onset preeclampsia. Am J Pathol 2015;185:1334-1343.

17. Grundberg I, Kiflemariam S, Mignardi M et al. In situ mutation detection and visualization of intratumor heterogeneity for cancer research and diagnostics. Oncotarget 2013;4:2407-2418.

18. Weibrecht I, Lundin E, Kiflemariam S et al. In situ detection of individual mRNA molecules and protein complexes or posttranslational modifications using padlock probes combined with the in situ proximity ligation assay. Nat Protoc 2013;8:355-372.

19. Hanna N, Hanna I, Hleb M et al. Gestational age-dependent expression of IL-10 and its receptor in human placental tissues and isolated cytotrophoblasts. J Immunol 2000;164:5721-5728.

20. Moussa M, Mognetti B, Dubanchet $S$ et al. Expression of beta chemokines in explants and trophoblasts from early and term human placentae. Am J Reprod Immunol 2001;46:309-317.

21. Naruse K, Innes BA, Bulmer JN et al. Secretion of cytokines by villous cytotrophoblast and extravillous trophoblast in the first trimester of human pregnancy. J Reprod Immunol 2010;86:148-150.

22. von Hundelshausen P, Petersen F, Brandt E. Platelet-derived chemokines in vascular biology. Thromb Haemost 2007;97:704-713.

23. Fraccaroli L, Alfieri J, Larocca $L$ et al. A potential tolerogenic immune mechanism in a trophoblast cell line through the activation of chemokine-induced $\mathrm{T}$ cell death and regulatory $\mathrm{T}$ cell modulation. Hum Reprod 2009;24:166-175.

24. Svinarich DM, Bitonti OM, Araneda $\mathrm{H}$ et al. Induction and postranslational expression of G-CSF and RANTES in a first trimester trophoblast cell line by lipopolysaccharide. Am J Reprod Immunol 1996;36:256-259.

25. Renaud SJ, Sullivan R, Graham CH. Tumour necrosis factor alpha stimulates the production of monocyte chemoattractants by extravillous trophoblast cells via differential activation of MAPK pathways. Placenta 2009;30:313-319.

26. Wegmann TG, Guilbert LJ. Immune signalling at the maternal-fetal interface and trophoblast differentiation. Dev Comp Immunol 1992;16: 425-430.

27. Jokhi PP, King A, Jubinsky PT et al. Demonstration of the low affinity alpha subunit of the granulocyte-macrophage colony-stimulating factor receptor (GM-CSF-R alpha) on human trophoblast and uterine cells. J Reprod Immunol 1994;26:147-164.

28. Garcia-Lloret MI, Morrish DW, Wegmann TG et al. Demonstration of functional cytokine-placental interactions: CSF-1 and GM-CSF stimulate human cytotrophoblast differentiation and peptide hormone secretion. Exp Cell Res 1994;214:46-54.

29. Bennett WA, Lagoo-Deenadayalan S, Whitworth NS et al. Expression and production of interleukin-10 by human trophoblast: relationship to pregnancy immunotolerance. Early Pregnancy 1997;3:190-198.

30. Moore KW, O'Garra A, de Waal Malefyt R et al. Interleukin-10. Annu Rev Immunol 1993;11:165-190.

31. Re F, Strominger JL. IL-10 released by concomitant TLR2 stimulation blocks the induction of a subset of Th1 cytokines that are specifically induced by TLR4 or TLR3 in human dendritic cells. J Immunol 2004;173: 7548-7555.

32. Thaxton JE, Sharma S. Interleukin-10: a multi-faceted agent of pregnancy. Am J Reprod Immunol 2010;63:482-491.

33. Roth I, Fisher SJ. IL-10 is an autocrine inhibitor of human placental cytotrophoblast MMP-9 production and invasion. Dev Biol 1999;205: 194-204.

34. Royle C, Lim S, Xu B et al. Effect of hypoxia and exogenous IL-10 on the pro-inflammatory cytokine TNF-alpha and the anti-angiogenic molecule soluble flt-1 in placental villous explants. Cytokine 2009;47:56-60.

35. Peterson RA. Regulatory T-cells: diverse phenotypes integral to immune homeostasis and suppression. Toxicol Pathol 2012;40:186-204.
36. Alijotas-Reig J, Llurba E, Gris JM. Potentiating maternal immune tolerance in pregnancy: a new challenging role for regulatory $T$ cells. Placenta 2014;35:241-248.

37. Teles A, Thuere C, Wafula PO et al. Origin of Foxp3(+) cells during pregnancy. Am J Clin Exp Immunol 2013;2:222-233.

38. Samstein RM, Josefowicz SZ, Arvey A et al. Extrathymic generation of regulatory $\mathrm{T}$ cells in placental mammals mitigates maternal-fetal conflict. Cell 2012;150:29-38.

39. Saito S, Nakashima A, Shima T et al. Th1/Th2/Th17 and regulatory T-cell paradigm in pregnancy. Am J Reprod Immunol 2010;63:601-610.

40. Guerin LR, Prins JR, Robertson SA. Regulatory T-cells and immune tolerance in pregnancy: a new target for infertility treatment? Hum Reprod Update 2009;15:517-535.

41. Morrish DW, Whitley GS, Cartwright JE et al. In vitro models to study trophoblast function and dysfunction- a workshop report. Placenta 2002;23:S114-S118.

42. Miller RK, Genbacev O, Turner MA et al. Human placental explants in culture: approaches and assessments. Placenta 2005;26:439-448.

43. Haider S, Knofler M. Human tumour necrosis factor: physiological and pathological roles in placenta and endometrium. Placenta 2009;30: 111-123.

44. Sainson RC, Johnston DA, Chu HC et al. TNF primes endothelial cells for angiogenic sprouting by inducing a tip cell phenotype. Blood 2008;111:4997-5007.

45. Castro Parodi M, Farina M, Dietrich V et al. Evidence for insulinmediated control of AQP9 expression in human placenta. Placenta 2011;32:1050-1056.

46. Bari MF, Weickert MO, Sivakumar K et al. Elevated soluble CD163 in gestational diabetes mellitus: secretion from human placenta and adipose tissue. PLoS One 2014;9:e101327.

47. Gauster M, Hiden U, van Poppel $M$ et al. Dysregulation of placental endothelial lipase in obese women with gestational diabetes mellitus. Diabetes 2011;60:2457-2464.

48. Lappas M, Yee K, Permezel M et al. Release and regulation of leptin, resistin and adiponectin from human placenta, fetal membranes, and maternal adipose tissue and skeletal muscle from normal and gestational diabetes mellitus-complicated pregnancies. J Endocrinol 2005;186:457-465.

49. Du MR, Wang SC, Li DJ. The integrative roles of chemokines at the maternal-fetal interface in early pregnancy. Cell Mol Immunol 2014;11: 438-448.

50. Chen SU, Chou CH, Chao KH et al. Lysophosphatidic acid up-regulates expression of growth-regulated oncogene-alpha, interleukin-8, and monocyte chemoattractant protein-1 in human first-trimester trophoblasts: possible roles in angiogenesis and immune regulation. Endocrinology 2010;151:369-379.

51. Bechi N, letta F, Romagnoli R et al. Environmental levels of paranonylphenol are able to affect cytokine secretion in human placenta. Environ Health Perspect 2010;118:427-431.

52. Ma Y, Mor G, Abrahams VM et al. Alterations in syncytiotrophoblast cytokine expression following treatment with lipopolysaccharide. Am J Reprod Immunol 2006;55:12-18.

53. Munro SK, Mitchell MD, Ponnampalam AP. Histone deacetylase inhibition by trichostatin A mitigates LPS induced TNFalpha and IL-10 production in human placental explants. Placenta 2013;34: 567-573.

54. Bayraktar $M$, Peltier $M$, Vetrano $A$ et al. IL-10 modulates placental responses to TLR ligands. Am J Reprod Immunol 2009;62: 390-399.

55. Koh YQ, Chan HW, Nitert MD et al. Differential response to lipopolysaccharide by JEG-3 and BeWo human choriocarcinoma cell lines. Eur J Obstet Gynecol Reprod Biol 2014;175:129-133. 